\title{
Circular RNA Profiling Reveals That circRNA_104433 Regulates Cell Growth by Targeting miR-497-5p in Gastric Cancer
}

This article was published in the following Dove Press journal: Cancer Management and Research

\author{
Weiyuan Wei ${ }^{1, *}$ \\ Xianwei Mol,* \\ Linhai Yan ${ }^{1} * *$ \\ Mingwei Huang' \\ Yang Yang' \\ Qinwen Jin' \\ Huage Zhong' \\ Wenlong $\mathrm{CaO}^{2}$ \\ Kun $\mathrm{Wu}^{2}$ \\ Liucheng Wu (iD) \\ Zhao $\mathrm{Li}^{1}$ \\ Tingan Wang' \\ Yuzhou Qin' \\ Jiansi Chen'
}

'Department of Gastrointestinal Surgery,

Affiliated Tumor Hospital, Guangxi

Medical University, Nanning 53002I,

People's Republic of China;

${ }^{2}$ Departments of Surgery, The First

Affiliated Hospital, Guangxi Medical

University, Nanning 53002I, People's

Republic of China

*These authors contributed equally to this work
Correspondence: Jiansi Chen; Yuzhou Qin Department of Gastrointestinal Surgery, Affiliated Tumor Hospital, Guangxi

Medical University, Nanning 53002I, People's Republic of China

Tel +86077I531042I

Email 2370860284@qq.com.cn;

qyz402@126.com
Background: The role and mechanism of hsa_circRNA_104433 in gastric cancer (GC) are further elucidated.

Materials and methods: CircRNA_104433 was selected by circRNA microarrays and GEO database. qRT-PCR was used to analyze the expression of circRNA_104433 in GC. The role of circRNA_104433 in GC cells was evaluated based on cell cycle progression, cell proliferation, cell apoptosis, and tumor xenograft experiment assay. To explore the mechanisms of circRNA_104433 in GC TCGA database, STRING version, qRT-PCR and luciferase assay were performed. Furthermore, the prognostic value of CDC25A in GC was determined based on the GEO database.

Results: The level of circRNA_104433 showed upregulation in GC tissues, and the expression of it showed a positive correlation with the degree of differentiation and the size of the tumor. Knockdown of circRNA_104433 inhibited cell cycle transition, and cell proliferation, while promoted cell apoptosis in GC. CircRNA_104433 directly binds to miR-497-5p, which directly regulates $\mathrm{CDC} 25 \mathrm{~A}$. The median survival period of GC patients with high expression levels of CDC25A was 21.3 months, which was shorter than those with low group expression of CDC25A (35.9 months). The cell cycle proteins CDK1, CDK2, CCNB1, PKMYT1, CDC20, CHEK1 and CDC25A were coexpressed with CDC25A.

Conclusion: These findings suggested that knockdown of circRNA_104433 expression suppressed tumor development in GC.

Keywords: circular RNA profiling, CircRNA_104433, miR-497-5p, CDC25A, gastric cancer, cell proliferation

\section{Introduction}

According to world health statistics, there are more than 750,000 new cases were diagnosed with gastric cancer (GC) each year globally. Although surgery, chemotherapy, radiotherapy and other comprehensive treatments are often used to treat GC patients, the therapeutic outcomes and clinical prognosis are still not satisfactory. The 5-year survival rate of GC patients is $25 \%$ to $35 \%$. Currently, there is a lack of comprehensive understanding regarding the pathogenesis of GC, as well as on specific GC tumor markers for clinical diagnosis. There are only a limited number of oncogenes and tumor suppressor genes described in GC till date. Therefore, it is imperative to study the mechanism of GC development, explore new biomarkers and therapeutic targets to facilitate early diagnosis and clinical prognosis of GC patients. 
With the advancements in medical research, new progressions were made in studying the transcription, translation, and coding of genes. Studies on circular RNA (circRNA) have brought new hope in the research of GC. circRNA is a new type of RNA that is different from traditional linear RNA, wherein it has a closed loop structure and is expressed in eukaryotic cells. In the transcriptome, most of the circular RNAs are composed of exon sequences, which are conserved in different species, and express specificity in different developmental stages of the tissues. In 2013, Hansen et al have found that the expression of ciRS-7 blocked miR-7, showing the function of this circular RNA. ${ }^{1,2}$ These circRNAs act as miRNA sponge in different species, and they are also called as competitive endogenous RNA (ceRNA) due to their competitive binding to miRNA. Hence, miRNA regulates the expression of target genes and is influenced by circular RNA that competitively binds to miRNA, and this in turn plays an important role in disease regulation.

The occurrence and development of GC involves multigene and multi-factoral interaction process. Previous studies have shown that circRNAs are associated with the occurrence and development of GC. Previous studies have shown that downregulation of circRNA_100269 is associated with poor prognosis in GC, while overexpression of circRNA_100269 inhibited miR-630 expression and tumor cell growth. Meanwhile, few other studies found that circRNA_0047905, circRNA_0138960, and circRNA_7690-15 were up-regulated in GC cells, while down-regulation of their expression inhibited cell proliferation and invasion of GC cells. However, the functional role and mechanism of circular RNAs in the development of GC should be fully elucidated.

Recent studies have revealed that miR-497-5p plays a tumor-suppressive role in GC, melanoma, head and neck squamous cell carcinoma, ${ }^{3-5}$ while it interacts with mismatch repair pathway and Wnt, RAS, MAPK, PI3K, TGF- $\beta$ and $p 53$ signaling pathways and influence the development of colorectal cancer. ${ }^{6} \mathrm{CDC} 25 \mathrm{~A}$ (also known as cell division cycle 25A) belongs to CDC25 family of phosphatases, which participates in cell cycle progression from G1 to $\mathrm{S}$ phase, and activates the cyclin-dependent kinase $\mathrm{CDC} 2$ by removing the two phosphate groups. CDC25A is specifically degraded in response to DNA damage, preventing cells with chromosomal abnormalities from progression through cell division. Thus, CDC25A is regarded as an oncogene, but its exact role in oncogenesis has not been demonstrated. ${ }^{7}$ Aberrant CDC25A expression was associated with disease progression in $\mathrm{GC}^{8}$, skin $^{\text {cancer }^{9}}$ and retinoblastoma. ${ }^{10}$ The expression of CDC25A is downregulated by miR-497 in chondrosarcoma. ${ }^{11}$ However, the interaction between circRNA, miR-497-5p and CDC25A in GC has not been reported till now.

Hence, in this study, we found that circRNA_104433 and CDC25A were abnormally overexpressed in GC, but miR497-5p expression was abnormally decreased in GC, showing a close relation with the development of GC. Downregulation of circRNA_104433 inhibited the growth of GC cells both in vitro and in vivo, and hsa_circRNA_104433/miR-497-5p/ CDC25A signaling pathway played an important role in this process. When the expression of hsa_circRNA_104433 was downregulated by lentiviral RNA interference, the expression of miR-497-5p was up-regulated. Furthermore, miR-497-5p directly regulated $\mathrm{CDC} 25 \mathrm{~A}$ signaling pathway and hsa_circRNA_104433 inhibited cell growth and promoted cell apoptosis in $\mathrm{GC}$, which in turn provide ideas for designing GC treatments.

\section{Materials and Methods Clinical Specimens}

Five pairs of gastric carcinoma tissues and corresponding nonmalignant tissues were obtained from participants by gastrectomy from The People's Hospital of Guangxi Zhuang Autonomous Region (Nanning, China) for circRNA chip detection. Additionally, 61 pairs of gastric carcinoma tissues and corresponding nonmalignant tissues were obtained from participants through gastrectomy from The First Affiliated Hospital of Guangxi Medical University (Nanning, China) for validation. Written informed consent was obtained from all patients before collecting the tissues. All samples were divided into two parts. Some specimens were immediately frozen in liquid nitrogen and stored at $-80^{\circ} \mathrm{C}$ until use, another set of specimens were fixed in 10\% formalin and analyzed by hematoxylin-eosin staining (HE). No other treatments should be received by patients before undergoing surgery. The subjects were informed about the study design and purpose according to the Declaration of Helsinki. This study was approved by the Ethics Committee of Affiliated Tumor Hospital of Guangxi Medical University. The pathological diagnoses were done according to the WHO 2007 classification of gastric carcinoma.

\section{Microarray Analysis}

Five pairs of gastric carcinoma tissues and their corresponding nonmalignant tissues were detected by circRNA microarray. The experiment and data collection were completed by 
Kang Cheng Biological Company, Shanghai, China. Labeled cRNAs were hybridized onto the Arraystar Human circRNA Array V2 (8x15K, Arraystar). The arrays were scanned by the Agilent Scanner G2505C. Agilent's feature extraction software (version 11.0.1.1) was used to analyze the acquired array images.

\section{Identification of circRNAs and Analysis of GEO DataSets}

For comparing the profile differences of the two groups (cancer versus paracancer), the "fold change" (i.e. the ratio of the group averages) between the groups for each circRNA was computed. Statistically significant difference was evaluated by $t$-test. CircRNAs with 2 fold changes and $P$-values of $<0.05$ were considered to be significantly different. The circRNA/ microRNA interaction was predicted using Arraystar's home-made miRNA target prediction software based on TargetScan ${ }^{12} \&$ miRanda, ${ }^{13}$ and the differentially expressed circRNAs in all comparisons were annotated in detail with circRNA/miRNA interaction information.

circRNAs expression profiles with accession GSE83521 and accession GSE93541 were obtained from the GEO DataSets. The significantly differentially expressed circRNAs with accession numbers GSE83521 and GSE93541 were obtained through bioinformatic analysis. Accession number GSE83521 had significantly different circRNAs expression profiles at stage III GC relative to normal mucosal tissue. The accession number GSE93541 contained significantly different circRNAs expression profiles for 6 human plasma samples derived from patients with GC and healthy control. The chip results of intersections of circRNAs, with accession numbers GSE83521 and GSE93541 were chosen. The most significantly different hsa_circRNA_104433 was chosen for conducting further experiments.

\section{Cell Culture}

The gastric cell lines GES, SGC-7901, HGC-27, MGC-803 and MGC-823 were obtained from Cell Bank of Shanghai Institute of Cell Biology, Chinese Academy of Sciences (Shanghai, China). The cells were maintained in RPMI 1640 medium (Gibco, USA) supplemented with 10\% fetal bovine serum (Gibco, USA), 100 units/mL penicillin, and $100 \mu \mathrm{g} / \mathrm{mL}$ streptomycin at $37^{\circ} \mathrm{C}$ and $5 \% \mathrm{CO}_{2}$.

\section{RNA Extraction and qRT-PCR}

Total RNA was extracted from fresh tissues and cells using TRIzol reagent (Invitrogen, Carlsbad, USA). Reverse transcription was performed using RNA PCR kit (AMV, TaKaRa). Quantitation of circRNA, miRNAs and mRNA were carried out by qRT-PCR using SYBR Premix Ex TaqTM (TaKaRa, Otsu, Japan) according to the manufacturer's instructions. U6 or GAPDH was used as an internal control for gene expression. Primer sequences are presented in Table 1. CircRNA_104433 expression level in $61 \mathrm{GC}$ tissues was calculated using $\Delta \mathrm{Ct}$ method with $\mathrm{GAPDH}$ as the control, and the lower $\Delta \mathrm{Ct}$ values indicate higher expression levels. The relative expression of each gene with the control group in cell line was calculated by 2- $\triangle \triangle \mathrm{Ct}$ method.

\section{Cell Transfection}

A lentiviral vector harboring RNAi sequence targeting human circRNA_104433 gene was constructed and identified by Genechem (Shanghai, China). The vector GV493 (hU6-MCS-CBh-gcGFP-IRES-puromycin) was used for lentiviral vector harboring RNAi. The transfection process and cell culturing were performed according to the lentiviral transfection manual as provided by Genechem (Shanghai, China). MGC-823 and SGC-7901 were transfected with lentiviral vectors as negative controls, and named as 823-NC and 7901-NC groups. MGC-823 and SGC-7901 were transfected with lentiviral vectors harboring RNAi sequence targeting hsa_circRNA_104433, and named as 823-Si- circRNA_104433 group and 7901- SicircRNA_104433 group. Cells were collected from each group after 72 hrs of transfection, which were then used in subsequent experiments.

\section{Cell Cycle Assay}

The GC cell lines, MGC-823 and SGC-7901, were collected after transfection at $72 \mathrm{hrs}$ and digested using routine methods. The cell suspensions were prepared with PBS and the cell concentration of each cell suspension was counted. After adjusting the number of cells to $1 \times 10^{6} /$ sample in a total volume of $1 \mathrm{~mL}$ in each group, $400 \mu \mathrm{L}$ propidium iodide (PI) was added to each sample, and then incubated at $4{ }^{\circ} \mathrm{C}$ in a refrigerator in darkness for $30 \mathrm{~min}$. The cell cycle of the cell samples were detected by flow cytometer after incubation for $30 \mathrm{~min}$.

\section{Cell Proliferation Assay}

The GC cell lines, MGC-823 and SGC-7901, were harvested after transfection at $72 \mathrm{hrs}$. $100 \mu \mathrm{L}$ of cell suspension containing 2000 cells was added to each well in a 96-well plate, and each group was run twice. Cell proliferation was detected 
Table I Base Sequence of Primers Used in This Work

\begin{tabular}{|l|l|}
\hline Name & Base Sequence \\
\hline miR-497-5p primer & 5'-CAGCAGCACACTGTGGTTTGTAA-3' \\
U6 forward primer & 5'- GGAACGATACAGAGAAGATTAGC-3' \\
U6 reverse primer & 5'- TGGAACGCTTCACGAATTTGCG-3' \\
circRNA_104433 forward primer & 5'-ATCCCAGAGGCTCTCCTCAC-3' \\
circRNA_104433 reverse primer & 5'-AGCACATCAAGAAGCCCATC-3' \\
CDC25A forward primer & GGCAAGCGTGTCATTGTTGTG \\
CDC25A reverse primer & ACAGCTCAGGGTAGTGGAGTTTGG \\
GAPDH forward primer & 5'-GTGCACCTTGGTCCATTTG-3' \\
GAPDH reverse primer & 5'-TGGTGAAGACGCCAGTGGA-3' \\
circRNA_104433-RNAi(70665-2) & 5'-CCGGCTTCAAGTGTCGAGATTGCCTCGAGGCAATCTCGACACTTGAAGTTTTT-3' \\
\hline
\end{tabular}

at 5-time points ( $0 \mathrm{~h}, 24 \mathrm{~h}, 48 \mathrm{~h}, 72 \mathrm{~h}$, and $96 \mathrm{~h})$. At each time point, $10 \%$ cck- 8 diluent was added to each well, and the absorbance of each well was measured at a wavelength of $450 \mathrm{~nm}$ by using a microplate reader.

\section{Cell Apoptosis Assay}

The GC cell lines, MGC-823 and SGC-7901, were collected after transfection for $72 \mathrm{hrs}$. The number of cells was diluted to $2 \times 10^{6}$ cells/sample in a total volume of $1 \mathrm{~mL}$ in each group. The sample was then subjected to centrifugation at $1000 \mathrm{rpm}$ for $5 \mathrm{~min}$, the supernatant was discarded, and the cells were re-suspended in $100 \mu \mathrm{L} 1 \mathrm{x}$ binding buffer. To each sample, $5 \mu \mathrm{L}$ Annexin V-PE and 7-AAD were added and incubated at room temperature in darkness for $30 \mathrm{~min}$. Cell apoptosis was detected by flow cytometry after incubation for $30 \mathrm{~min}$.

\section{AO-EB Double Staining and Hoechst Assay}

The GC cell lines, MGC-823 and SGC-7901, were collected after transfection for $72 \mathrm{hrs}$. The number of cells in each group was adjusted to $4 \times 10^{5}$ cells $/ \mathrm{mL}$. Next, $60 \mu \mathrm{L}$ of diluted cell suspension was added into 24-well plate and then cultured in a cell culture box for $24 \mathrm{~h}$.

For AO-EB double staining assay, AO solution and EB solution were mixed at a ratio of $1: 1$ to prepare a working fluid. Then, $20 \mu \mathrm{L}$ of the working fluid was added directly into per milliliter of the medium of the well. Cell apoptosis was determined by fluorescence microscopy after incubation for $5 \mathrm{~min}$ at room temperature.

For Hoechst assay, $500 \mu \mathrm{L}$ of Hoechst 33258 staining solution was added into each well, with shaking for $5 \mathrm{~min}$. Cell nuclei apoptosis was detected by fluorescence microscopy at $350 \mathrm{~nm}$ excitation wavelength and at $460 \mathrm{~nm}$ emission wave length.

\section{Tumor Xenograft Experiment}

All animal studies were approved by The Ethics Committee of Affiliated Tumor Hospital of Guangxi Medical University. The laboratory and animal care were provided according to the protocols stipulated by the experimental animal care and use guidelines of our hospital. Male athymic nude BALBC/c mice of about 4-5 weeks old were used. The GC cell line MGC-823 was collected after transfection for $72 \mathrm{hrs}$. Then, each medial axilla of each mouse was hypodermically injected with a dose of $5 \times 10^{6}$ cells in $250 \mu \mathrm{L}$ PBS. Each group had 4 nude mice, in which 823-NC group was injected with negative control lentiviral vectors transfected into MGC-823 cells and 823-Si-circRNA_104433 group was injected with lentiviral vectors with RNAi sequence targeting hsa_circRNA_104433 transfected into MGC-823 cells. The length and width of subcutaneous transplantation were measured every 4 days for 24 days using the formula, $\mathrm{V}=$ tumor size, using the formula; $\mathrm{V}\left(\mathrm{mm}^{3}\right)=$ length $\times($ width $/ 2){ }^{2}$ After 24 days, the mice were euthanized and the volume of tumors was assessed.

\section{Prediction of miRNA and circRNA}

\section{Interactions}

To identify the target microRNAs for hsa_circRNA_104433, the hsa_circRNA_104433/microRNA interaction was predicted using the Arraystar's home-made miRNA target prediction software based on TargetScan \& miRanda. The data containing original microarray data of miRNAs in 446 GC tissues and 45 normal gastric tissues were also obtained from The CancerGenome Atlas (TCGA) database. The R language (Version R3.44) edgeR package was used to analyze the differences in the expression of miRNAs, and the differentially expressed miRNAs were screened using the filter 
condition of $\log 2$ FoldChange $>1$ and $\mathrm{P} \quad$ value $<0.05$. According to the nature of interactions between circRNA and miRNA, the miRNAs that show opposite expression in GC in TCGA database were chosen for further analysis. The intersection of the above data included candidate miRNAs, which in turn could be the target genes of hsa_circRNA_104433.

\section{Prediction of miRNA and mRNA Interactions}

To identify the target mRNA for hsa-miR-497-5p, the data were predicted using 3 websites and the following algorithms were used: TargetScan (https://www.targetscan. org), miRTarBase (http://mirtarbase.mbc.nctu.edu.tw) and miRDB (http://www.mirdb.org). The intersections of the 3 databases were chosen for further analysis. Data containing original microarray data of mRNAs in 375 GC tissues and 32 normal gastric tissues were also obtained from TCGA database. The R language (Version R3.44) edgeR package was used to analyze the differences in expression of mRNAs, and differentially expressed mRNAs were screened out using the filter condition of $\log 2$ FoldChange $>1$ and $P$ value of $<0.05$. According to the nature of interaction between miRNA and mRNA, the mRNAs that show the opposite expression in GC in TCGA database were chosen for further analysis. The intersection of the two datasets was considered as the candidate mRNAs, which act as target genes for hsa-miR-497-5p. In addition, the co-expression of mRNA and hsa-miR-497$5 p$ in GC was analyzed, and the expression data on cancers were downloaded from the TCGA project via Genomic Data Commons Data Portal. To analyze the proteins encoded by the mRNA targeted by miR-497-5p, the functions of the proteins were analyzed using the online tool STRING database (https://string-db.org, version 10.5).

\section{Dual Luciferase Reporter Assay}

To confirm the relationship among hsa_circRNA_104433, miR-497-5p, and CDC25A, dual luciferase reporter assay was performed. The luciferase activities were measured using the dual-luciferase reporter assay system (Promega, Madison, WI). Before plasmid transfection, the isolated cells were cultured on 24-well plates for $24 \mathrm{hrs}$. To determine the success of transfection after $24 \mathrm{hrs}$, the fluorescence level of the GFP marker gene was observed by fluorescence microscopy. The dual-luciferase ${ }^{\circledR}$ reporter assay system (promega E1910) kit was used to prepare the cells and detect the luciferase activity.

\section{Western Blot Assay}

GAPDH and CDC25A rabbit anti-human antibodies were purchased from Cell Signaling Technology. CCNB1 rabbit anti-human antibodies were bought from Abcam. Anti-rabbit antibodies for GAPDH, CDC25A, and CCNB1 and secondary antibodies of IRDye 800 raised in goat were purchased from Li-Cor Biosciences (Lincoln, NE, USA). The proteins were extracted from cells using Western blot and IP kits (Beyotime, Beijing, China) and protease inhibitor phenylmethanesulfonyl fluoride (Beyotime, Beijing, China). Protein concentration was detected by enhanced BCA protein assay kit (Beyotime, Beijing, China). The Western blot procedure was performed as described in our previous publication. ${ }^{14}$ The membranes of the protein blots were scanned by Odyssey Software Version 3.0 system (Li-Cor Biosciences Lincoln, NE, USA). GAPDH protein was used as an internal reference to calculate the expression of each protein.

\section{Statistical Analysis}

Statistical data were analyzed using SPSS 17.0. The data are presented as means $\pm \mathrm{SD}$. Student's $t$-test and a chi-squared test were used for statistical analysis. For each statistical test, $P<0.05$ was regarded as significant difference.

\section{Results}

\section{Expression Profile of Circular RNA in GC}

There are 5 gastric cancer and 5 adjacent normal gastric tissues in the circRNA expression profiles. After normalizing the intensity values of all samples, the median of difference of samples was normalized almost on the same line as shown in the box plot (Figure 1A). The variations in circRNAs expression across different samples were evaluated by scatterplot visualization (Figure 1B). Differentially expressed circRNAs with statistical significance between the two groups were identified through volcano plot filtering (Figure 1C), and they were constructed using fold-change values and $P$-values. Based on differentially expressed circRNAs, the hierarchical clustering was established to describe the differences in circRNA expression among the samples (Figure 1D). A total of 2758 circRNAs showed significant aberrations in the expressions between the two groups $(P<0.05$, Table S1). With a cut-off of fold-change value $\geq 2.0$ and $P$-value $\leq 0.05$, a total of 683 circRNAs showed differential expression based on microarray analysis, in which 352 were upregulated and 331 were down-regulated (Table S1). 

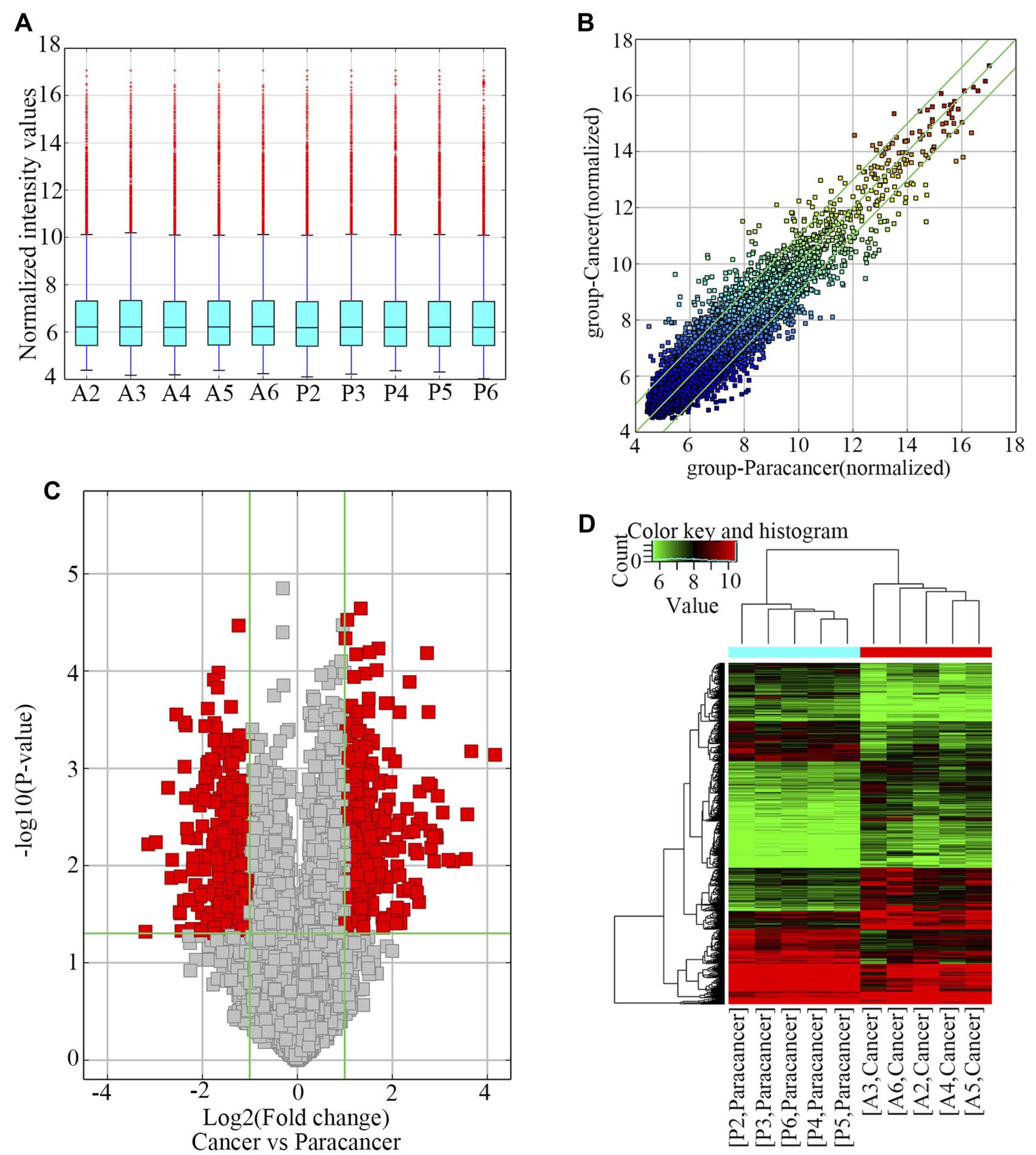

D
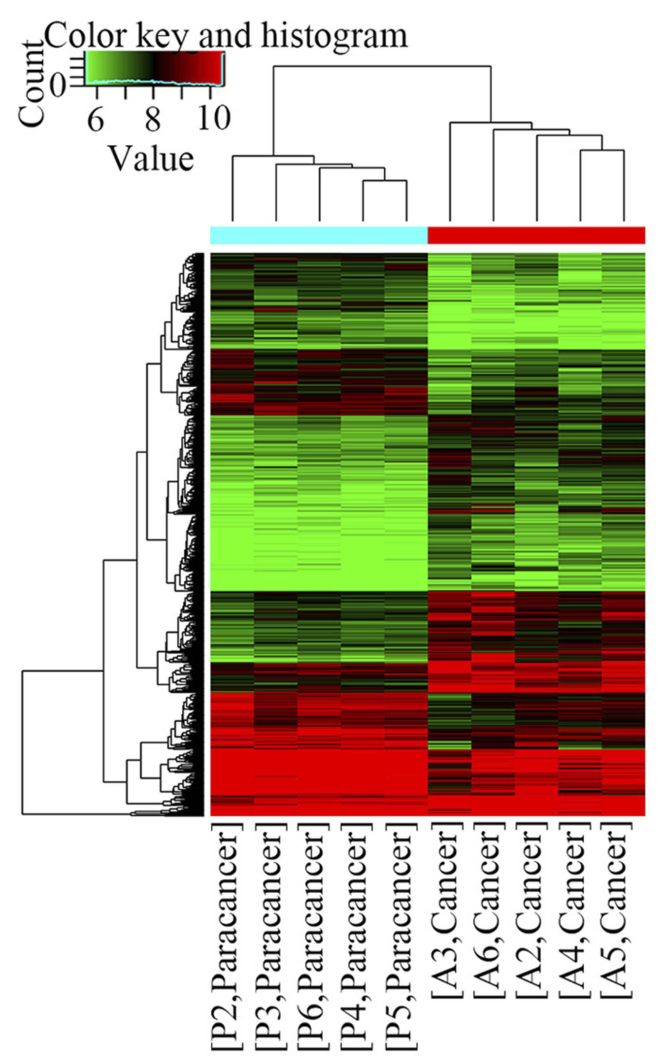

Figure I Differences in circRNA expression profile between GC tissues and paired adjacent normal tissues.

Notes: (A) The box plot showed variations in circRNA expression. (B) Scatter plots are used to evaluate the differences in circRNA expression. (C) Volcano plots illustrated differential circRNA expression, in which the red points represent the differentially expressed circRNAs with statistical significance. (D) The heatmap shows differentially expressed circRNAs in 5 GC tissues and paired adjacent normal tissues. Gene expression profiles are presented in rows. "Red" indicates high relative expression, and "blue" indicates low relative expression.

\section{Annotation of Different circRNAs in GC} The general characteristics of data from 683 circRNAs were analyzed. The chromosome location, classification type, length, and fold-change distribution of circRNAs are presented in supplementary Table S1. The binding sites for miRNA and the names of miRNAs are shown 
in Table S1. Most of the circRNAs are located on chromosome 1. The length of different circRNAs ranged between $\leq 500 \mathrm{nt}$ and 4001 16000nt. The fold-changes of all circRNAs ranged from 2 to 2.5. The circRNAs are classified into 5 types: "exonic", "intronic", "antisense", "sense overlapping" and "intergenic". The functional classification of circRNAs mainly focused on the exonic type.

\section{The Intersection of Different Chip Data}

Data regarding the expression profiles of circRNAs, with accession numbers GSE83521 and GSE93541 were obtained from the GEO DataSets. The expression of these circRNAs was analyzed by bioinformatic tools. A total of 3071 circRNAs showed significant differential expression between the 2 groups in the microarray of GSE83521 $(P<0.05$, Table S2), and 1618 of these were up-regulated and 1453 were down-regulated (Table S2). A total of 621 circRNAs showed differential expression between the 2 groups based on the microarray analysis of GSE93541 ( $P<0.05$, Table S2), wherein 332 were up-regulated and 289 were down-regulated (Table S2). Intersections of circRNA of different expressions of our chip results (fold-change value $\geq 2.0$ and $P$-value $\leq 0.05$ ), with accession numbers GSE83521 and GSE93541 are shown in Table 2. A total of 9 circRNAs showed upregulation and 5 showed downregulation (Figure 2A and B). Among these, hsa_circRNA_104433 showed the highest up-regulation, with a Log2 (fold change) of 4.426 .

\section{Up-Regulation of hsa_circRNA_I04433 in $\mathrm{GC}$}

The results of qRT-PCR showed that hsa_circRNA_104433 was up-regulated in SGC-7901, HGC-27, MGC-803 and MGC-823 cell lines when compared to normal cell line GES $(P<0.05$, Figure 2C). Compared to normal adjacent tissues from 61 patients, hsa_circRNA_104433 showed upregulation in GC tissues $(P<0.05$, Figure 2D), and its expression was positively correlated with the degree of differentiation and size of the tumor $(P<0.05)$. The expression of circRNA_104433 showed no correlation with T classification, gender or age $(P$ $>0.05$, Table 3 ). Compared with negative control group, the expression of hsa_circRNA_104433 was down-regulated in gastric cells that were transfected with lentiviral vectors harboring RNAi sequence targeting hsa_circRNA_104433 $(P<0.05$, Figure 2E).

\section{Knockdown of hsa_circRNA_I04433 Inhibited GC Proliferation}

The results of flow cytometry showed that the percentage of cell phase G0/G1 in Si- circRNA_104433 group was increased and the percentage of cell phase $\mathrm{S}$ in $\mathrm{Si}$ circRNA_104433 group was decreased when compared to the negative control group $(P<0.05$, Figure $3 \mathrm{~A}$ and $\mathrm{B})$. This indicated that down-regulation of hsa_circRNA_104433 prevented cells from entering the $\mathrm{S}$ phase from $\mathrm{G} 1$ in MGC-823 and SGC-7901 cells, suppressing cell division and proliferation. The results of CCK-8 analysis showed that the A value

Table 2 The Differently Expressed circRNAs Between Gastric Cancer and Normal Gastric Tissues

\begin{tabular}{|l|l|l|l|l|l|l|l|l|}
\hline CircRNA & P value & Log2 FC & Regu & Catalog & Chrom & Strand & Best Transcript & Gene Symbol \\
\hline hsa_circRNA_104433 & 0.006 & 4.426 & Up & Exonic & chr7 & + & NM_005720 & ARPCIB \\
hsa_circRNA_102I2I & 0.006 & 4.230 & Up & Exonic & chrl7 & - & NM_000088 & COLIAI \\
hsa_circRNA_102400 & 0.046 & 2.498 & Up & Exonic & chrl9 & + & NM_004368 & CNN2 \\
hsa_circRNA_104084 & 0.017 & 2.321 & Up & Exonic & chr6 & + & NR_0154I0 & CASCI5 \\
hsa_circRNA_101904 & 0.016 & 2.302 & Up & Exonic & chrl6 & + & NM_017869 & BANP \\
hsa_circRNA_101826 & 0.006 & 2.248 & Up & Exonic & chrl6 & + & NM_003869 & CES2 \\
hsa_circRNA_10189I & 0.033 & 2.209 & Up & Exonic & chrl6 & + & NM_03I476 & CRISPLD2 \\
hsa_circRNA_10154I & 0.003 & 2.133 & Up & Exonic & chrl5 & - & NM_004039 & ANXA2 \\
hsa_circRNA_101539 & 0.003 & 2.107 & Up & Exonic & chrl5 & - & NM_004039 & ANXA2 \\
hsa_circRNA_10004I & 0.027 & 2.638 & Down & Exonic & chrl & - & NM_012102 & RERE \\
hsa_circRNA_102374 & 0.046 & 2.264 & Down & Exonic & chrl8 & + & NM_015285 & WDR7 \\
hsa_circRNA_400102 & $<0.001$ & 2.190 & Down & Intronic & chr9 & - & ENS298532 & SNAPC4 \\
hsa_circRNA_101525 & 0.021 & 2.185 & Down & Exonic & chrl5 & + & NM_004855 & PIGB \\
hsa_circRNA_102924 & 0.005 & 2.096 & Down & Exonic & chr2 & - & NM_006216 & SERPINE2 \\
\hline
\end{tabular}

Abbreviations: CircRNA, circular RNA; FC, fold change; Regu, regulation; chrom, chromosome. 
A

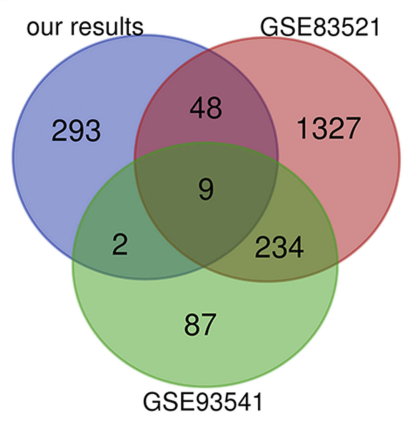

B

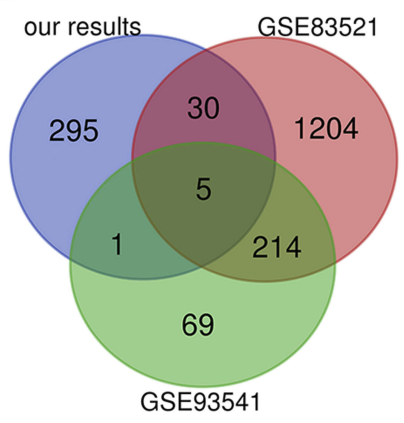

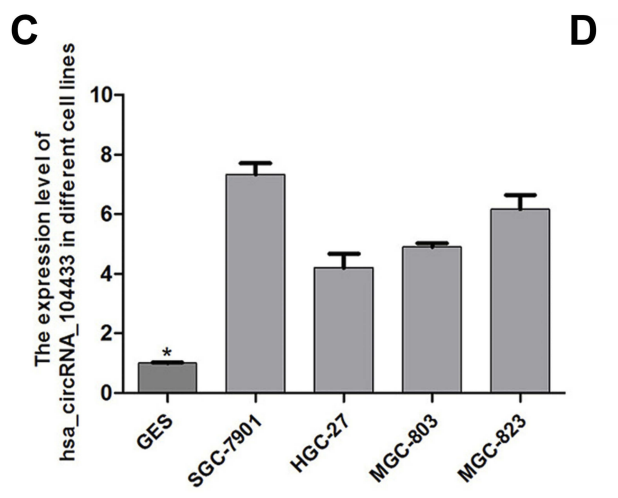

D

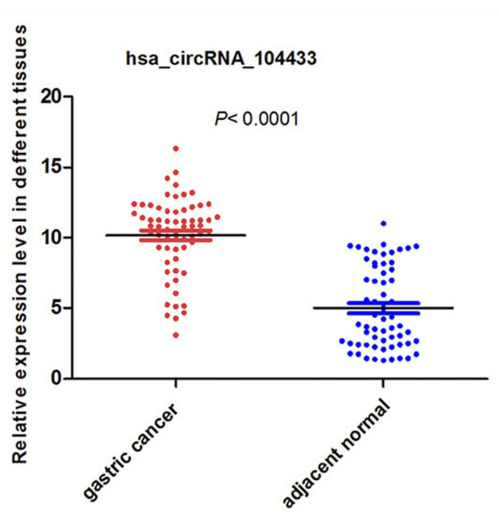

E

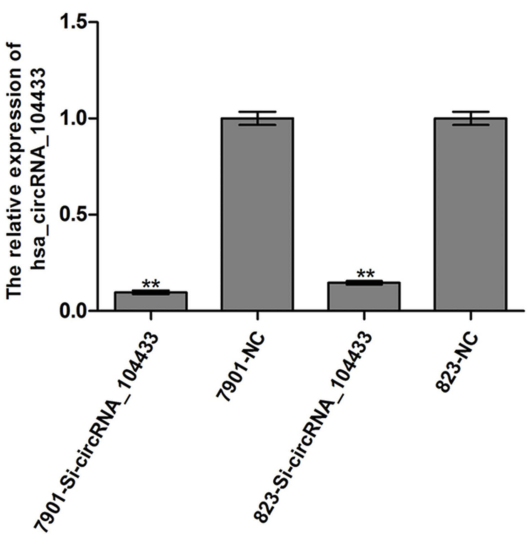

Figure 2 Filtering and validation of differentially expressed circRNAs in GC.

Notes: (A, B) Based on differentially expressed circRNAs of our chip results, GEO DataSets were selected using Venn diagram. (C-E) qRT-PCR verified the expression of circRNA_104433 in GC cell lines, in which 6I GC patients and GC cells transfected with lentiviral vectors showed knockdown of circRNA_104433. All the data are presented as mean \pm S.D. $* P<0.05$, $* * P<0.01$.

of Si- circRNA_104433 group was lower than that of the negative control group in MGC-823 and SGC-7901 cells after transfection for $72 \mathrm{~h}(P<0.05)$, and these results indicated that down-regulation of hsa_circRNA_104433 prevented cell proliferation (Figure 3D and E).

\section{Knockdown of hsa_circRNA_I04433 Promoted GC Cell Apoptosis}

Compared with negative control group, the apoptotic rate of GC cells was higher in 823-Si- circRNA_104433 group and 7901-Si- circRNA_104433 group than that in the negative control groups (Figure 4A-F). These results indicated that down-regulation of hsa_circRNA_104433 promoted cell apoptosis in MGC-823 and SGC-7901 cells.

\section{Knockdown of hsa_circRNA_104433 Inhibited GC Growth in vivo}

To further explore the function of circRNA_104433 in tumor growth in vivo, xenograft experiments were performed, and one nude BALBC/c mouse died naturally in each group during the experiment. The volume of tumors was smaller in 823-Si- circRNA_104433 group and 7901- SicircRNA_104433 group than that in the negative control groups (Figure $4 \mathrm{G}$ and $\mathrm{H}$ ). These results indicated that knockdown of circRNA_104433 effectively inhibited tumor growth in GC.

\section{The Interaction Between Differentially Expressed circRNAs and miRNAs}

The circRNA/microRNA interaction was predicted using the Arraystar's home-made miRNA target prediction software, and the differentially expressed circRNAs across the groups were annotated in detail using the circRNA/miRNA interaction information. Each circRNA was predicted to bind to 5 possible microRNAs, and the names of the microRNAs are provided in the column "MREs" of the excel file that contained the differentially expressed circRNAs (Table S1). 
Table 3 Clinico-Pathological Variables and the Expression Leve $(\triangle \mathrm{ct})$ of circRNA_104433 in Gastric Cancer Patients

\begin{tabular}{|c|c|c|c|}
\hline Characteristics & $\begin{array}{l}\text { No. of Cases } \\
\text { (\%) }\end{array}$ & Mean $\pm S D$ & $P$ value \\
\hline \multicolumn{4}{|l|}{ Age (years) } \\
\hline$<60$ & $37(60.7)$ & $4.604 \pm 0.47 \mid$ & 0.178 \\
\hline$\geq 60$ & $24(39.3)$ & $5.635 \pm 0.98$ & \\
\hline \multicolumn{4}{|l|}{ Gender } \\
\hline Male & $42(68.9)$ & $4.885 \pm 0.45 I$ & 0.623 \\
\hline Woman & $19(31.1)$ & $5.285 \pm 0.675$ & \\
\hline \multicolumn{4}{|l|}{ Tumor size $(\mathrm{cm})$} \\
\hline$<5$ & $32(52.46)$ & $5.015 \pm 0.532$ & $0.043^{*}$ \\
\hline$\geq 5$ & $29(47.54)$ & $4.222 \pm 0.487$ & \\
\hline \multicolumn{4}{|l|}{ TNM stage } \\
\hline I-II & $25(40.98)$ & $4.23 I \pm 0.555$ & 0.081 \\
\hline III-IV & $36(59.02)$ & $5.550 \pm 0.485$ & \\
\hline \multicolumn{4}{|l|}{ Lymph node metastasis } \\
\hline No & $21(34.43)$ & $4.372 \pm 0.639$ & 0.218 \\
\hline Yes & $40(65.57)$ & $5.344 \pm 0.455$ & \\
\hline \multicolumn{4}{|l|}{ Distant metastasis } \\
\hline Mo & $42(68.85)$ & $4.972 \pm 0.484$ & 0.883 \\
\hline MI & $19(31.15)$ & $5.092 \pm 0.554$ & \\
\hline \multicolumn{4}{|l|}{ Differentiation } \\
\hline Well and moderate & $13(2 \mid .31)$ & $6.987 \pm 0.736$ & $0.005^{*}$ \\
\hline Poor & $48(78.69)$ & $4.474 \pm 0.399$ & \\
\hline
\end{tabular}

Note: * Statistically significant.

Abbreviation: TNM, tumor, node, metastasis.

\section{Construction of circRNA-miRNA-mRNA Regulation Network}

The target mRNAs of hsa-miR-497-5p, hsa-miR-545-3p, hsa-miR-636, hsa-miR-15a-5p, hsa-miR-335-3p were predicted by TargetScan, miRTarBase and miRDB, and the results showed that the total number of target mRNAs of hsa-miR-497-5p/ hsa-miR-545-3p/ hsa-miR-636/ hsa-miR15a-5p/hsa-miR-335-3p are 27, 64, 16, 42 and 58, respectively (Table S2).

\section{miR-497-5p Is Negatively Regulated by hsa_circRNA_104433}

According to the analysis of TCGA database that contained the original microarray data of miRNAs from $375 \mathrm{GC}$ tissues and 32 normal gastric tissues, differentially expressed miRNAs were screened out, and the filter condition was $\log 2$ FoldChange $>1$ and $P$ value $<0.05$. A total of 305 differentially expressed miRNAs were screened out from TCGA database, wherein 208 miRNAs showed up-regulation and
97 miRNAs showed down-regulation (Table S2). The circRNA/microRNA interaction was predicted using the Arraystar's home-made miRNA target prediction software, and the results showed that the 5 miRNAs that interacted with hsa_circRNA_104433 included hsa-miR-497-5p, hsamiR-545-3p, hsa-miR-636, hsa-miR-15a-5p and hsa-miR335-3p. According to the nature of interaction between circRNA and miRNA, the down-regulated miRNAs in GC based on TCGA database were chosen further as an intersection object. The gene found at the intersection of the 2 data was hsa-miR-497-5p, which was considered as the target gene of hsa_circRNA_104433. This was further confirmed by biluciferase report experimental data, in which the relative luciferase activity of circRNA WT + miRNA group was lower than that of NC group and circRNA MT+ miRNA group $(P<0.05$, Figure $5 \mathrm{~A}$ and $\mathrm{C})$. Compared with control vector group cells, the levels of hsa-miR-497-5p were higher in 823-Si- circRNA_104433 group and 7901- SicircRNA_104433 group $(P<0.05$, Figure 5E). These data suggested that low expression of hsa_circRNA_104433 effectively increased the expression of hsa-miR-497-5p in MGC-803 and SGC-7901 cell lines in vitro. Thus, it is considered that hsa_circRNA_104433 can reduce hsa-miR497-5p expression as knockdown of circRNA_104433 resulted in the up-regulation of hsa-miR-497-5p.

\section{CDC25A Was Negatively Regulated by hsa-miR-497-5p}

According to the original microarray data of mRNAs from $375 \mathrm{GC}$ tissues and 32 normal gastric tissues in TCGA database, 11,557 differentially expressed mRNAs were identified, in which 7921 mRNAs showed up-regulation and 3636 mRNAs showed down-regulation (Table S2). The target genes of hsa-miR-497-5p were predicted by TargetScan, miRTarBase and miRDB databases, and the intersections of the 3 databases were chosen. A total of 27 genes were identified (Table S2), and their functions were analyzed using the online tool STRING version 10.5. According to the nature of interaction between miRNA and mRNA, the up-regulated mRNAs in GC from TCGA database were used as subsequent intersection objects. CDC25A was found to be the target gene of hsa-miR-497-5p. According to the analysis of original microarray data from TCGA database on $372 \mathrm{GC}$ tissues, the expression levels of hsa-miR-497-5p and CDC25A showed negative correlation $(P<0.05)$. The data indicated that CDC25A might be a target gene of hsa-miR497-5p in GC tissues. This was further confirmed by 

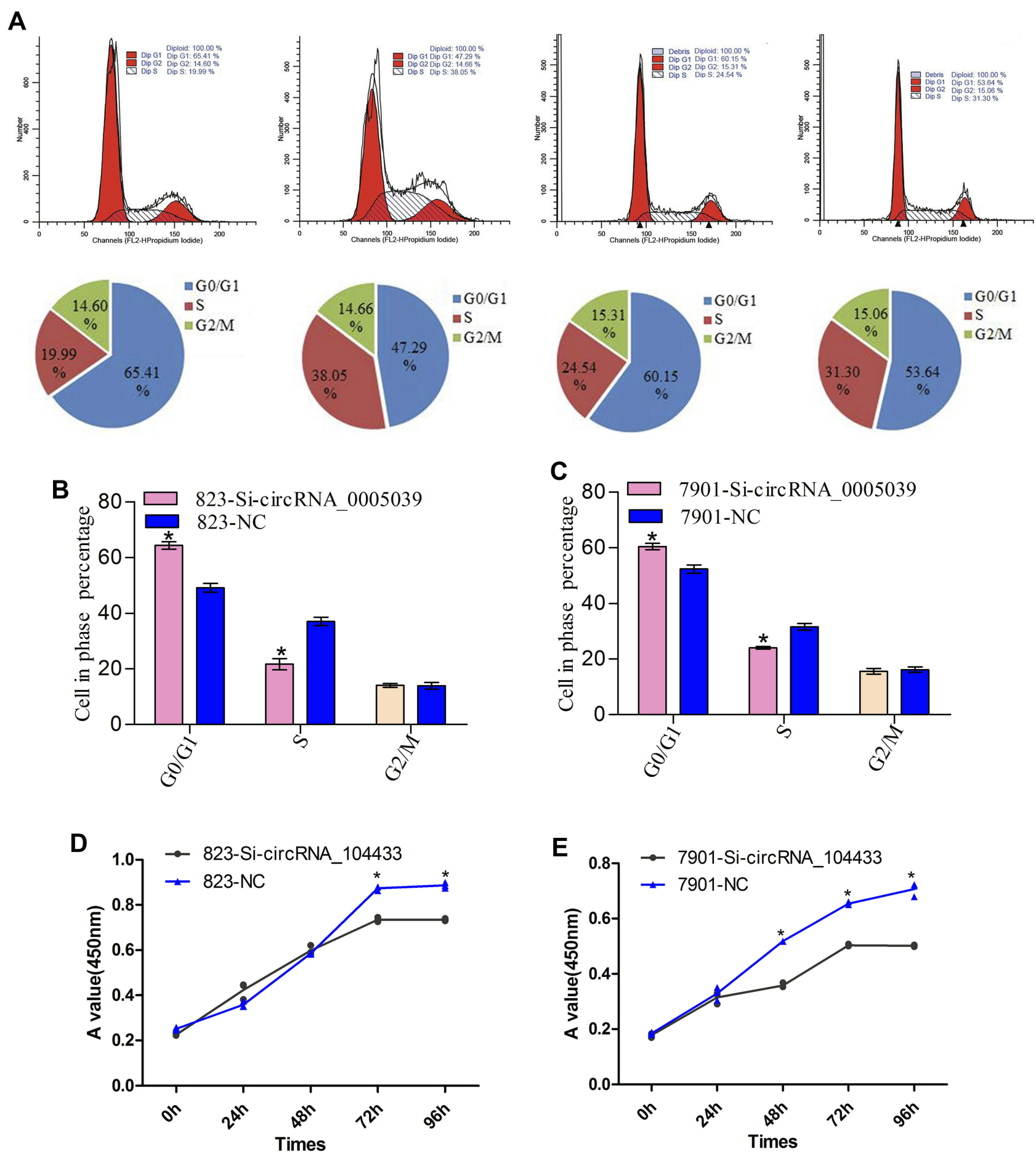

Figure 3 The effect of knockdown of circRNA_104433 on cell cycle distribution and proliferation in GC cells.

Notes: (A) Flow cytometer assay was performed to assess the cell cycle. (B, C) The cell cycle distribution of each group was compared. (D, E) CCK8 assay was performed to assess cell proliferation. The OA value curve was used to compare cell proliferation of each group. All data are presented as mean \pm S.D. $* P<0.05$.

biluciferase reporter experiment, wherein the relative luciferase activity of CDC25A 3' UTR WT+miRNA group was lower than that of NC group and CDC25A 3' UTR MT both
+ miRNA group $(P<0.05$, Figure 5B and D). Meanwhile, knockdown of circRNA_104433 resulted in the up-regulation of hsa-miR-497-5p and down-regulation of CDC25A 


\section{MGC-823}
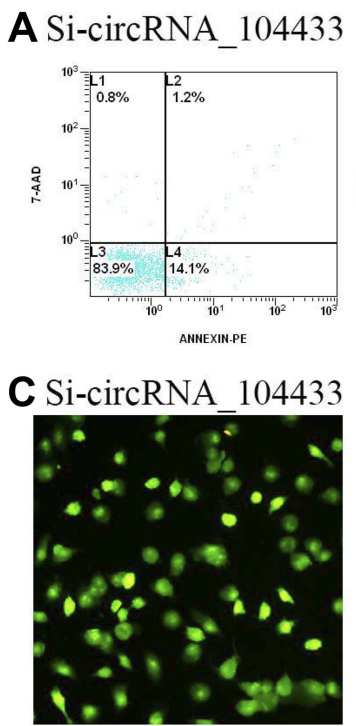

E Si-circRNA 104433

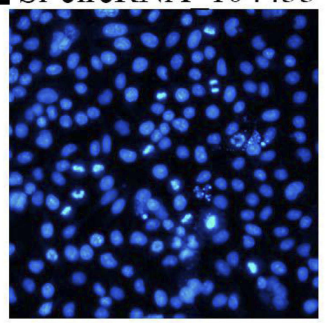

G
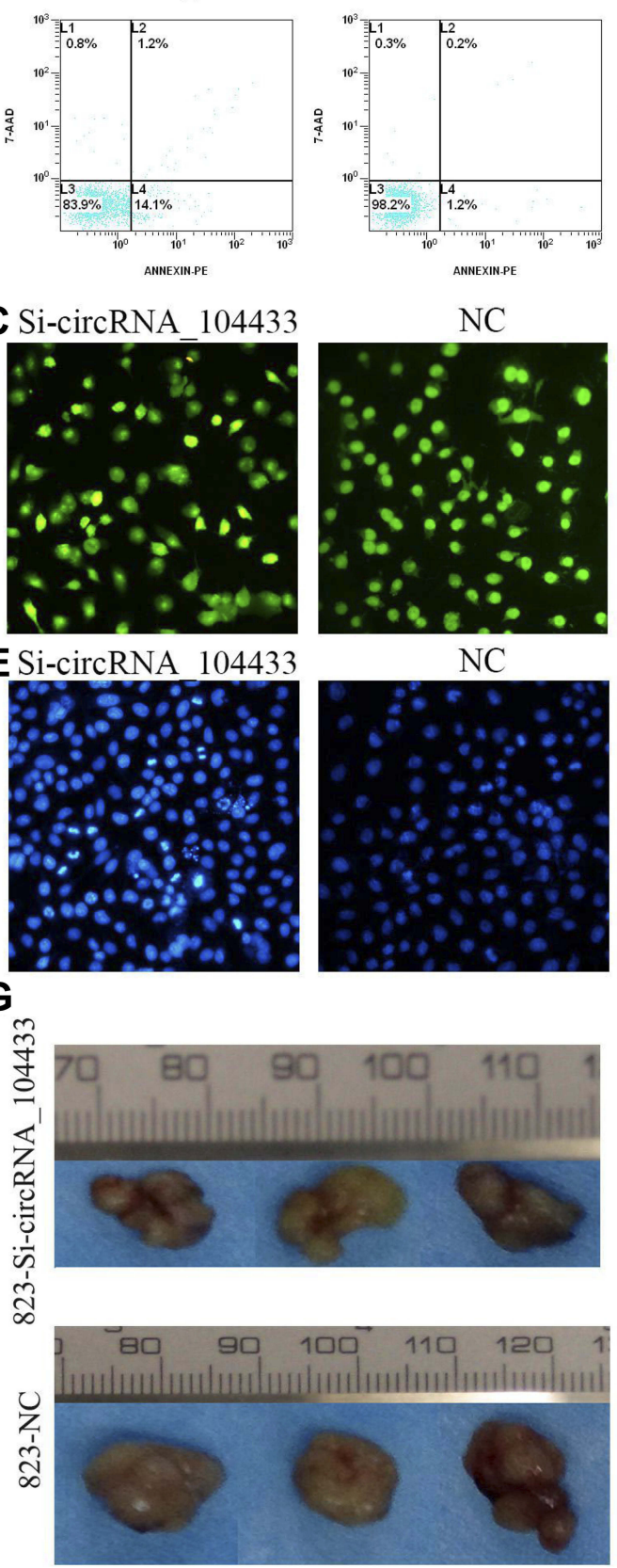

SGC-7901
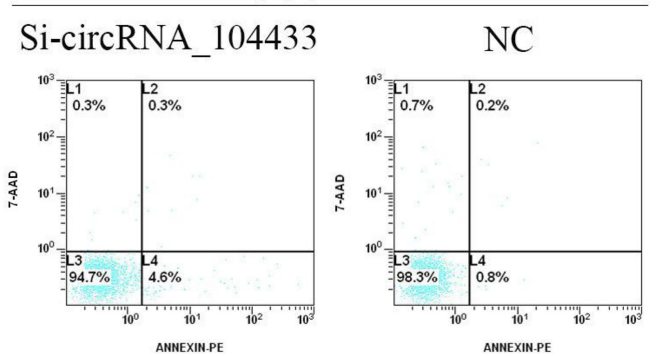

B

Si-circRNA 104433
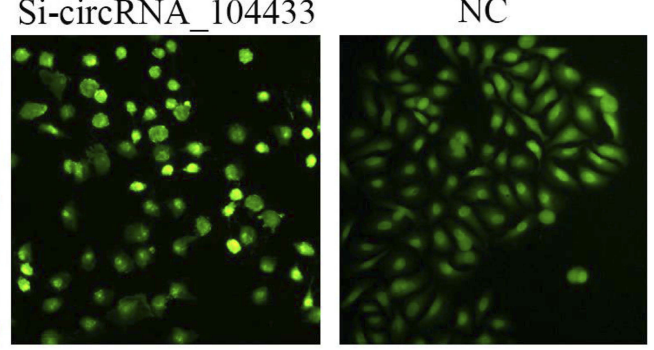

$\mathrm{NC}$
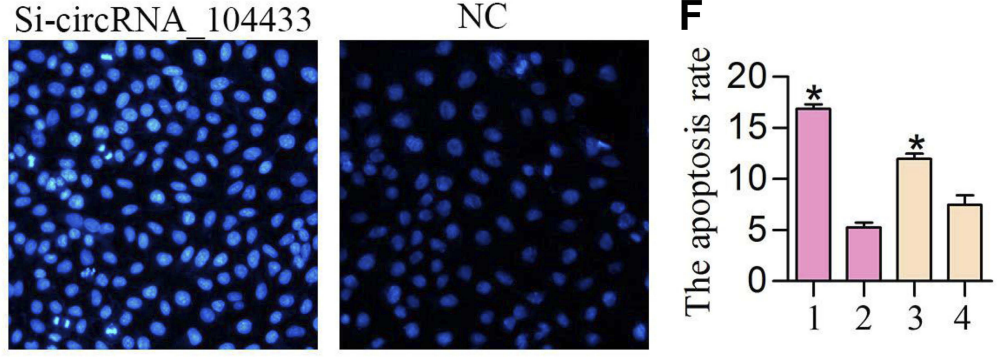

H

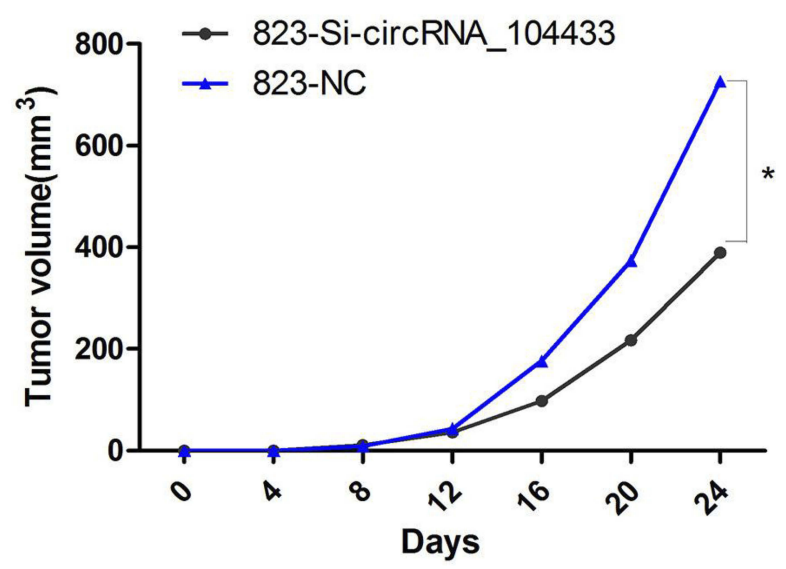

Figure 4 The effect of knockdown of circRNA_104433 on cell apoptosis and tumor growth of xenograft in GC.

Notes: (A, C, E) Flow cytometer assay, AO-EB double staining and Hoechst assay were performed to assess cell apoptosis, respectively. (B, D, F) The rate of cell apoptosis of each group was compared. (G) The images of transplanted tumors in each group (three per group) in nude mice. (H) The growth curve was used to compare the tumors of each group. The tumor volume was measured every four days. All data are presented as mean \pm S.D. $* P<0.05$.

and CCNB1 in 823-Si- circRNA_104433 group and 7901Si- circRNA_104433 group when compared with control vector group cells $(P<0.05$, Figures $5 \mathrm{E}-\mathrm{F}$ and $6 \mathrm{~A})$. These results indicated that hsa-miR-497-5p can reduce the expression of CDC25A.

\section{Prognostic Value and Coexpression Gene Analysis of CDC25A in GC}

The network proteins associated with $\mathrm{CDC} 25 \mathrm{~A}$ were analyzed using the online tool STRING version 10.5 . The proteins CDC25A, CDK1, CDK2, CCNB1, PKMYT1, CDC20 
A hsa_circRNA_104433 WT(387-407) 5'...CACCCCAACAATGTGCTGCTG...3'

hsa-miR-497-5p

3'...UGUUUGGUGUCACACGACGAC...5'

hsa_circRNA_104433 MT(387-407) 5'...CACCCCAACAATCACGACGAG...3'

B CDC25A 3' UTR WT( 365-371) 5'...GAAGUUACACAGAAAUGCUGCUG...3'

hsa-miR-497-5p

3'...UGUUUGGUGUCACACGACGAC...5'

CDC25A 3' UTR MT( 365-371) 5'...GAAGUUACACAGAAAUGCACGAG...3'

CDC25A 3' UTR WT( 674-681) 5'...CUGUGGUACUGGGGCUGCUGCUA....3'

hsa-miR-497-5p

3'...UGUUUGGUGUCACACGACGAC...5'

CDC25A 3' UTR MT( 674-681) 5'...CUGUGGUACUGGGGCACGACGAA...3'

C

D
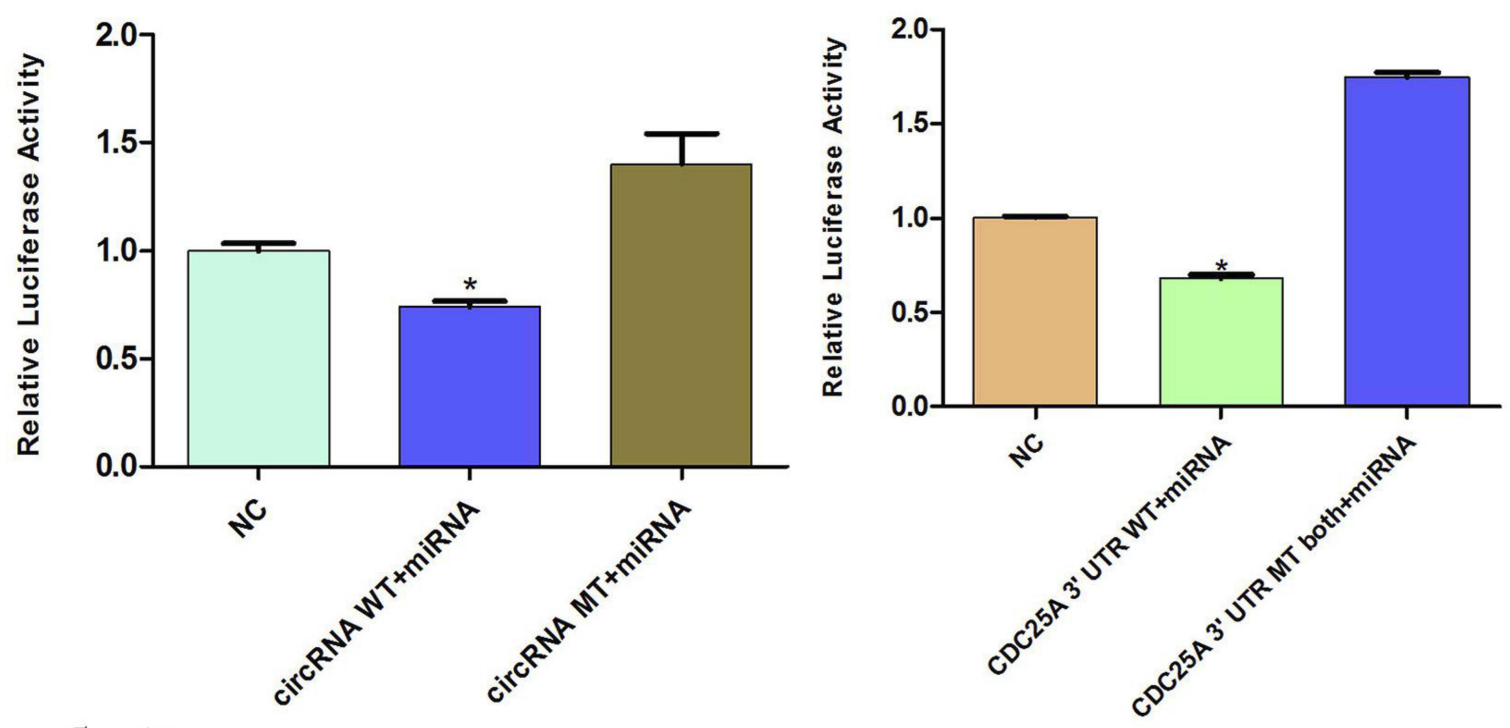

$\mathbf{E}$
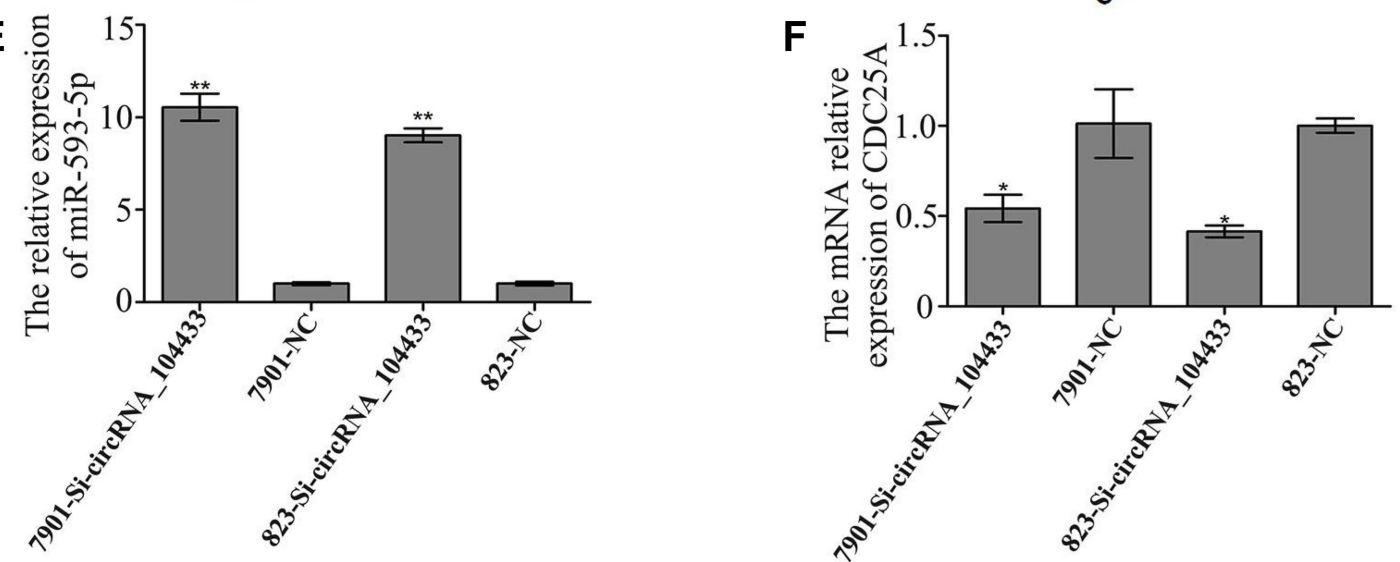

Figure 5 Confirmation of target gene.

Notes: (A) The base sequence of interaction of miR-497-5p and circRNA_104433 wild-type 3'-UTR and continuous missing style of 3' UTR reporters. (B) The base sequence of interaction of miR-497-5p and CDC25A wild-type 3'-UTR and continuous missing style of 3'UTR reporters. (C, D) Comparison of differentially expressed luciferase activity in each group. (E, F) Comparison of differentially expressed miR-497-5p and CDC25A in each group. All data are presented as mean \pm S.D. $* P<0.05$, $* * P<0.01$. 
A
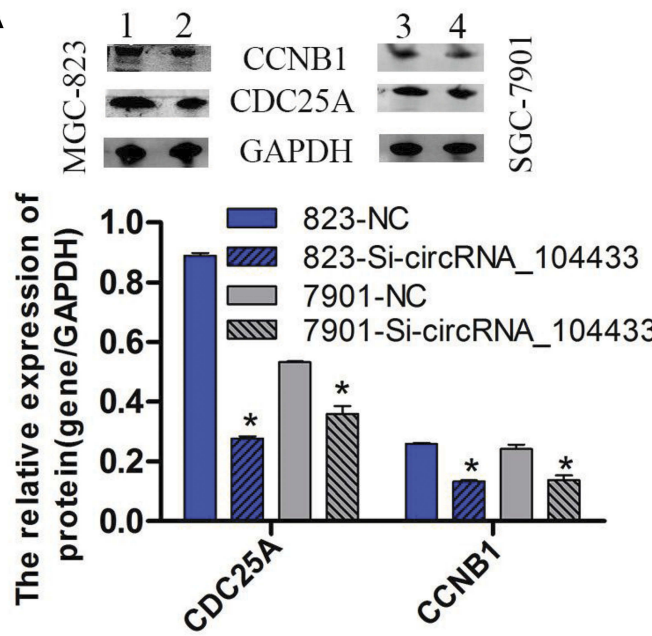

C Network nodes represent proteins Node Color

Colored nodes: query proteins and first shell of interactors

Pathway description

Mitotic nuclear divsion: Function in network by Biological Process (GO)

Cell cycle : Function in network by KEGG Pathways

Node Content

Filled nodes: some 3D structure is known or predicted
B

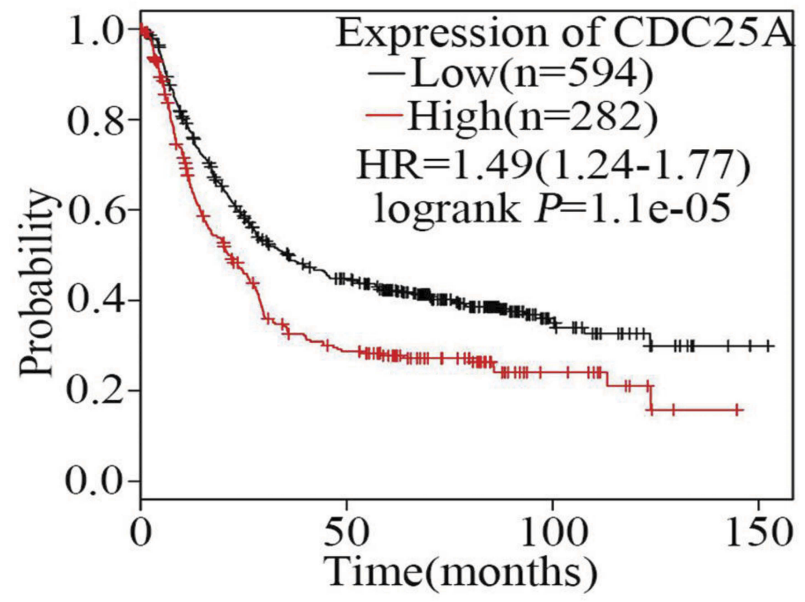

Edges

Edges represent protein-protein associations Known Interactions

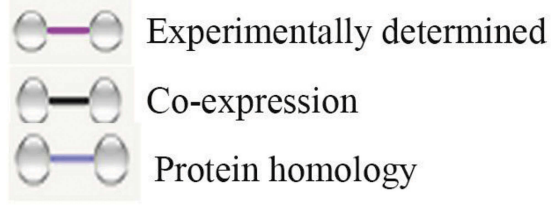

Edge Confidence

Action effects

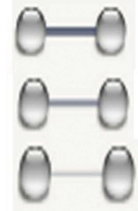

Highest (0.900)

High (0.700)

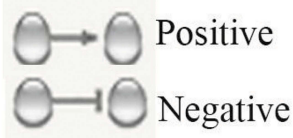

Medium (0.400)
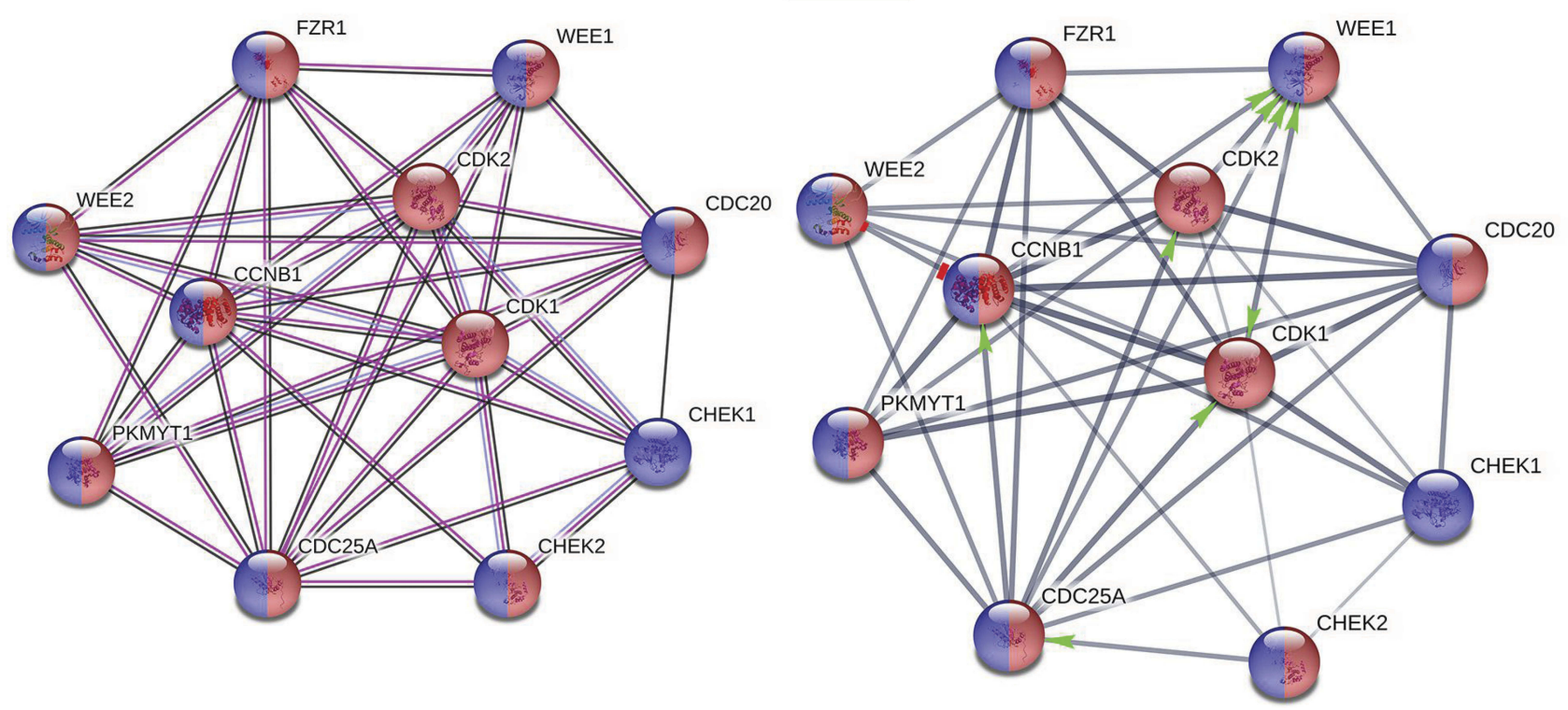

Figure 6 The prognosis of CDC25A and its related protein mechanism in GC cells.

Notes: (A) Images of genes and protein expressions were detected by Western blotting assay. Comparison of differentially expressed genes in each group. I, 823-NC group; 2, 823-Si- circRNA 104433 group; 3, 790I-NC group, 4, 790I-Si- circRNA 104433 group. All data are presented as mean \pm S.D. *P < 0.05 . (B) The Kaplan Meier plot was performed to assess the effect of CDC25A gene expression on survival rate in $876 \mathrm{GC}$ patients. Analysis of original microarray data on GEO DataSets with original microarray data (GSEI4210, GSEI5459, GSE22377, GSE29272, GSE5I I05, GSE62254). (C) The network proteins associated with CDC25A were analyzed using the online tool STRING version 10.5 . 
WEE2, WEE1 and CHEK1 showed interaction with CDC25A. The proteins CDK1, CDK2, CCNB1, PKMYT1, CDC20, CHEK1 and CDC25A were coexpressed in homo sapiens, in which they regulate the cell cycle. Additionally, some of these were involved in mitotic nuclear division as shown in Figure 6C. The Kaplan Meier plotter was used to assess the effect of 54,675 genes on the survival rate in 1065 GC patients, with a mean follow-up period of 33 months. According to the analysis of original microarray data on GEO DataSets (GSE14210, GSE15459, GSE22377, GSE29272, GSE51105, GSE62254), the number of patients with high expression of CDC25A included 282, and those with low expression of CDC25A included 594. The median survival period of patients with high expression levels of CDC25A (21.3 months) was shorter than that of those with low expression of CDC25A (35.9 months) $(P<0.05$, Figure 6B).

\section{Discussion}

GC is prevalent in middle- and late-stage individuals. Most of the GC patients are unsuitable for surgical treatment due to advanced stages, while a few others do not respond to chemotherapy. However, individualized genetargeting therapy is considered to be an effective method for destroying tumors and in turn can find the diseasecausing genes in such patients. So, regulation of gene expression is one approach for inhibiting tumor cells. Therefore, finding new strategies in the field of GC to improve the therapeutic efficacy is an important proposal.

CircRNAs are special class of non-coding RNAs, which are regarded as emerging research hotspots in the field of RNA. CircRNA is different from the traditional linear RNA (containing 5 "and 3" ends), wherein the molecules of circRNA have a closed circular structure and are not affected by RNA exonucleases, and the expression of circRNA remains more stable and not easy to be degraded. ${ }^{15,16}$ MicroRNAs and long intergenic noncoding RNAs (lincRNAs) were extensively studied and reported in GC. However, there were only a few studies on the role of circRNAs in GC. Therefore, this study was conducted to determine the role of circRNAs in $\mathrm{GC}$, and the results revealed that many circRNAs showed abnormal expression in GC cells based on microarray analysis. Combined with GEO database analysis, circRNA_104433 showed abnormally high expression in GC tissues and in the sera of GC patients. Further experimental results showed that circRNA_104433 was highly expressed in GC cell lines, and positively correlated with the degree of cell differentiation and size of the tumor. Moreover, knockdown of circRNA_104433 inhibited tumor growth and promoted cell apoptosis in GC. These results showed that circRNA_104433 plays a crucial role in GC development and might act as a potential therapeutic target and a biomarker for GC.

MicroRNAs play an important role in a variety of diseases. Shan et al have reported that miR-497-5p was upregulated in ApoE-deficient mice and this might play an important role in the pathogenesis of atherosclerosis. ${ }^{17}$ Overexpression of hsa-miR-497-5p causes negative regulation of SMAD gene in HEK293t cells and results in cell cycle arrest, ${ }^{18}$ as well as suppresses tumor growth and invasion in angiosarcoma and melanoma. ${ }^{4,19}$ During therapy, elevated levels of extracellular microvesicle miR-497-5p lead to prolonged progression-free survival in metastatic cutaneous malignant melanoma. ${ }^{20}$ According to TCGA database analysis, hsa-miR-497-5p was shown to be down-regulated in GC. Liu et al have reported that knockdown of LINC00662 reduced miR-497-5p expression and decreased GC cell proliferation, while increased the chemo-sensitivity of GC cells. ${ }^{3}$ Due to the crucial role of miR-497-5p in cancer, development of miR-497-5p-based gene therapy is of great significance for GC treatment.

Recently, several studies have reported that decreased CDC25A expression might inhibit malignant progression by suppressing cell-cycle pathway. ${ }^{7,21,22}$ Similarly, CDC25A plays a unique role in activating cyclin B1Cdk1 (CCNB1-CDK1) at the centrosome, ${ }^{23}$ and is thought to be regulated by microRNA-99a-5p, miR-497 and miR483-3p in cancer. ${ }^{11,24,25}$ According to TCGA database analysis, CDC25A showed upregulation in GC, while down-regulation of CDC25A prolonged the survival period in GC. Meanwhile, Guo et al have reported the involvement of PTEN-Akt-p53-miR-365-cyclin D1/CDC25A axis in gastric tumorigenesis, ${ }^{26}$ and the delayed downregulation of $\mathrm{CDC} 25 \mathrm{~A}$ might contribute to cell adaptation to quiescent state in GC. ${ }^{8}$ Meanwhile, proteins associated with cell cycle and mitotic nuclear division, such as CDK1, CDK2, CCNB1, PKMYT1, CDC20, CHEK1 and CDC25A were coexpressed with CDC25A. Therefore, $\mathrm{CDC} 25 \mathrm{~A}$ is considered to play an important role in cancer, and thus development of CDC25A-based gene therapy for GC treatment should be taken into consideration.

Numerous studies have confirmed that circRNAs acts as a miRNA sponge in different cancer types. ${ }^{27-29}$ In this study, hsa_circRNA_104433 reduced hsa-miR-497-5p expression through bioinformatics analyses, biluciferase report 
experiment, and circRNA_104433 knockdown experiments. Also, knockdown of circRNA_104433 resulted in downregulation of CDC25A and CCNB1. Several studies have confirmed that the functions of miRNA depend on their downstream target genes, as miRNA binds to $3^{\prime}$ UTRs of transcripts of target genes. ${ }^{30}$ In addition, bioinformatics analyses, real-time quantitative $\mathrm{PCR}$ and biluciferase report experiments confirmed that CDC25A was a target gene of hsa-miR-497-5p. Finally, theoretical and experimental findings of this study showed that circRNA_104433 and CDC25A are linked by miR-497-5p.

In conclusion, our study showed that circRNA_104433 is up-regulated in GC. Knockdown of circRNA_104433 inhibited tumor growth and promoted cell apoptosis in GC. CircRNA_104433 and CDC25A are linked by miR-497-5p. Downregulation of circRNA_104433 inhibited tumor progression in GC. Thus, circRNA_104433 is considered to play an important role in the development of therapies and might be used as a diagnostic biomarker for GC.

\section{Acknowledgments}

This work was supported by grants from the Natural Science Foundation of Guangxi (NO. 2018GXNSFB A138016), the National Natural Science Foundation of China (NO. 81660511), the Natural Science Foundation of Guangxi (NO.2015GXNSFDA227001), the Guangxi Medical University Training Program for Distinguished Young Scholars and Guangxi Clinical Research Center for Colorectal Cancer.

\section{Disclosure}

The authors declare that they have no competing interests in this work.

\section{References}

1. Hansen TB, Jensen TI, Clausen BH, et al. Natural RNA circles function as efficient microRNA sponges. Nature. 2013;495(7441):384 388. doi:10.1038/nature11993

2. Hansen TB, Kjems J, Damgaard CK. Circular RNA and miR-7 in cancer. Cancer Res. 2013;73(18):5609-5612. doi:10.1158/0008-5472. CAN-13-1568

3. Liu Z, Yao Y, Huang S, et al. LINC00662 promotes gastric cancer cell growth by modulating the Hippo-YAP1 pathway. Biochem Biophys Res Commun. 2018;505(3):843-849. doi:10.1016/j.bbrc.2018.09.191

4. Chai L, Kang XJ, Sun ZZ, et al. MiR-497-5p, miR-195-5p and miR-455-3p function as tumor suppressors by targeting hTERT in melanoma A375 cells. Cancer Manag Res. 2018;10:989-1003. doi:10.2147/CMAR. S163335

5. Verduci L, Ferraiuolo M, Sacconi A, et al. The oncogenic role of circPVT1 in head and neck squamous cell carcinoma is mediated through the mutant $\mathrm{p} 53 / \mathrm{YAP} / \mathrm{TEAD}$ transcription-competent complex. Genome Biol. 2017;18(1):237. doi:10.1186/s13059-017-1368-y
6. Falzone L, Scola L, Zanghi A, et al. Integrated analysis of colorectal cancer microRNA datasets: identification of microRNAs associated with tumor development. Aging (Albany NY). 2018;10(5):1000-1014. doi:10.18632/aging.v10i5

7. Biswas SC, Sanphui P, Chatterjee N, et al. Cdc25A phosphatase: a key cell cycle protein that regulates neuron death in disease and development. Cell Death Dis. 2017;8(3):e2692. doi:10.1038/cddis. 2017.115

8. Kang SH, Bang YJ, Jong HS, et al. Rapid induction of p21WAF1 but delayed down-regulation of Cdc25A in the TGF-beta-induced cell cycle arrest of gastric carcinoma cells. $B r J$ Cancer. 1999;80 (8):1144-1149. doi:10.1038/sj.bjc.6690478

9. Al-Matouq J, Holmes T, Hammiller B, et al. Accumulation of cytoplasmic CDC25A in cutaneous squamous cell carcinoma leads to a dependency on CDC25A for cancer cell survival and tumor growth. Cancer Lett. 2017;410:41-49. doi:10.1016/j.canlet.2017.09.023

10. Singh L, Pushker N, Sen S, et al. Expression of CDC25A and $\mathrm{CDC} 25 \mathrm{~B}$ phosphatase proteins in human retinoblastoma and its correlation with clinicopathological parameters. $\mathrm{Br} J$ Ophthalmol. 2015;99(4):457-463. doi:10.1136/bjophthalmol-2014-305830

11. Lu Y, Li F, Xu T, et al. miRNA-497 negatively regulates the growth and motility of chondrosarcoma cells by targeting Cdc25A. Oncol Res. 2016;23(4):155-163. doi:10.3727/096504016X14519157902681

12. Enright AJ, John B, Gaul U, et al. MicroRNA targets in drosophila. Genome Biol. 2003;5(1):R1. doi:10.1186/gb-2003-5-1-r1

13. Pasquinelli AE. MicroRNAs and their targets: recognition, regulation and an emerging reciprocal relationship. Nat Rev Genet. 2012;13 (4):271-282. doi: $10.1038 / \operatorname{nrg} 3162$

14. Yu H, Wei W, Cao W, et al. Regulation of cell proliferation and metastasis by microRNA-593-5p in human gastric cancer. Onco Targets Ther. 2018;11:7429-7440. doi:10.2147/OTT.S178151

15. Chen LL, Yang L. Regulation of circRNA biogenesis. RNA Biol. 2015;12(4):381-388. doi:10.1080/15476286.2015.1020271

16. Lukiw WJ. Circular RNA (circRNA) in Alzheimer's disease (AD). Front Genet. 2013;4(307). doi:10.3389/fgene.2013.00307

17. Shan Z, Yao C, Li ZL, et al. Differentially expressed microRNAs at different stages of atherosclerosis in ApoE-deficient mice. Chin Med $J$ (Engl). 2013;126(3):515-520.

18. Jafarzadeh M, Soltani BM, Dokanehiifard S, et al. Experimental evidences for hsa-miR-497-5p as a negative regulator of SMAD3 gene expression. Gene. 2016;586(2):216-221. doi:10.1016/j. gene.2016.04.003

19. Chen Y, Kuang D, Zhao X, et al. miR-497-5p inhibits cell proliferation and invasion by targeting $\mathrm{KCa} 3.1$ in angiosarcoma. Oncotarget. 2016;7(36):58148-58161. doi:10.18632/oncotarget.11252

20. Svedman FC, Lohcharoenkal W, Bottai M, et al. Extracellular microvesicle microRNAs as predictive biomarkers for targeted therapy in metastastic cutaneous malignant melanoma. PLoS ONE. 2018;13(11): e0206942. doi:10.1371/journal.pone.0206942

21. Sadeghi H, Golalipour M, Yamchi A, et al. CDC25A pathway toward tumorigenesis: molecular targets of $\mathrm{CDC} 25 \mathrm{~A}$ in cell-cycle regulation. J Cell Biochem. 2018;120(3):2919-28.

22. Biswas K, Philip S, Yadav A, et al. BRE/BRCC45 regulates CDC25A stability by recruiting USP7 in response to DNA damage. Nat Commun. 2018;9(1):537. doi:10.1038/s41467-018-03020-6

23. Lindqvist A, Kallstrom H, Lundgren A, et al. Cdc25B cooperates with $\mathrm{Cdc} 25 \mathrm{~A}$ to induce mitosis but has a unique role in activating cyclin B1-Cdk1 at the centrosome. J Cell Biol. 2005;171(1):35-45. doi: $10.1083 /$ jcb. 200503066

24. Qin H, Liu W. MicroRNA-99a-5p suppresses breast cancer progression and cell-cycle pathway through downregulating CDC25A. J Cell Physiol. 2018;234(4):3526-3537.

25. Bertero T, Gastaldi C, Bourget-Ponzio I, et al. CDC25A targeting by miR-483-3p decreases CCND-CDK4/6 assembly and contributes to cell cycle arrest. Cell Death Differ. 2013;20(6):800-811. doi:10.1038/ cdd. 2013.5 
26. Guo SL, Ye H, Teng Y, et al. Akt-p53-miR-365-cyclin D1/cdc25A axis contributes to gastric tumorigenesis induced by PTEN deficiency. Nat Commun. 2013;4(2544). doi:10.1038/ncomms3544.

27. Wang X, Fang L. Advances in circular RNAs and their roles in breast cancer. J Exp Clin Cancer Res. 2018;37(1):206. doi:10.1186/s13046-0180870-8

28. Belousova EA, Filipenko ML, Kushlinskii NE. Circular RNA: new regulatory molecules. Bull Exp Biol Med. 2018;164(6):803-815. doi:10.1007/s10517-018-4084-z
29. Yamamura S, Imai-Sumida M, Tanaka Y, et al. Interaction and crosstalk between non-coding RNAs. Cell Mol Life Sci. 2018;75(3):467484. doi:10.1007/s00018-017-2626-6

30. Qin Q, Furong W, Baosheng L. Multiple functions of hypoxia-regulated miR-210 in cancer. J Exp Clin Cancer Res. 2014;33:50. doi:10.1186/1756-9966-33-50

\section{Publish your work in this journal}

Cancer Management and Research is an international, peer-reviewed open access journal focusing on cancer research and the optimal use of preventative and integrated treatment interventions to achieve improved outcomes, enhanced survival and quality of life for the cancer patient.
The manuscript management system is completely online and includes a very quick and fair peer-review system, which is all easy to use. Visit http://www.dovepress.com/testimonials.php to read real quotes from published authors. 